\title{
LA DESCOMPENSACIÓN ENTRE LAS HABILIDADES DE CARÁCTER GRAMATICAL Y PRAGMÁTICO EN EL DISGURSO ORAL DE NIÑOS CON SÍNDROME DE ASPERGER
}

\author{
FRANCISCO J. RODRÍGUEZ MUÑOZ \\ Universidad de Almería
}

\section{RESUMEN}

El presente estudio parte de la premisa de que las habilidades lingüísticas de carácter gramatical que intervienen en la producción del discurso oral de los niños con síndrome de Asperger se muestran intactas o inalteradas, mientras que la mayor parte de las disfunciones en el plano discursivo se asocian a las habilidades específicamente pragmáticas. Esta idea es compatible con la teoría de la infraconectividad, de acuerdo con la cual existiría un correlato neurofisiológico capaz de explicar tales discrepancias entre las habilidades gramaticales y las pragmáticas. Asimismo, se propone que las disfunciones comunicativas en el síndrome de Asperger pueden deberse a un déficit de integración. Con objeto de apoyar los supuestos anteriores, se llevó a cabo el análisis de una serie de categorías que remiten, por un lado, a las habilidades de carácter gramatical (complejidad sintáctica y diversidad léxica, cohesión y coherencia) y, por otro, a las de carácter pragmático (principio de cooperación y tareas de edición). Para ello, se tuvieron en cuenta cuarenta grabaciones transcritas en formato CHAT (Codes for the Human Analysis of Transcripts); veinte procedían de hablantes con síndrome de Asperger, y otras veinte, de hablantes sin este trastorno. Los resultados de los análisis refuerzan el punto de partida de este trabajo, según el cual se producirían claras disonancias entre las habilidades estructurales del lenguaje y las comunicativas. Finalmente, con la ambición de alcanzar una mayor comprensión de los déficits comunicativos, particularmente, sobre el síndrome de Asperger, en esta investigación se busca la conexión interdisciplinar.

PALABRAS CLAVE: síndrome de Asperger, déficit comunicativo, déficit pragmático, déficit de integración, teoría de la infraconectividad.

\section{ABSTRACT}

The present study starts from the premise that the linguistic skills of grammatical nature involved in speech production of children with Asperger syndrome are shown intact or unaltered, whereas most of the dysfunctions in the discursive 
dimension are associated to specifically pragmatic skills. This idea is compatible with the underconnectivity theory, according to which there would be a neurophysiological correlate capable of explaining such discrepancies between grammatical and pragmatic skills. Likewise, it is considered that communicative dysfunctions in Asperger syndrome may be due to an integration deficit. In order to support these assumptions, the analysis of different categories is carried out; more exactly, grammatical skills (syntactic complexity and lexical diversity, cohesion and coherence) are compared to pragmatic skills (principle of cooperation and editing tasks). For this, forty recordings transcribed in CHAT format (Codes for the $\mathrm{Hu}-$ man Analysis of Transcripts) were taken into account. Twenty of them came from speakers with Asperger syndrome, and the other twenty, from speakers without this disorder. The results of this analysis reinforce the starting point of the paper, according to which there would be clear dissonances between the structural abilities of language and the communicative ones. Finally, with the ambition to reach a greater comprehension about communication disorders, particularly about Asperger syndrome, this research seeks an interdisciplinary connection.

KEYWORDS: Asperger syndrome, communication disorder, pragmatic deficit, integration deficit, underconnectivity theory.

\section{INTRODUCCIÓN}

Como señala Fernández Pérez (2014), el enfoque pragmático-comunicativo ha contribuido extraordinariamente tanto a exaltar el valor de las producciones verbales espontáneas como a otorgarle un papel protagonista a la denominada lingüística de corpus. Los datos procedentes de tales producciones son, por ejemplo, los que les han permitido a los lingüistas establecer perfiles en los que, además de describir el comportamiento comunicativo típico (regular) o atípico (peculiar) de los hablantes, se detectan áreas o habilidades más o menos problemáticas. Es tal el impacto que han tenido las investigaciones basadas en corpus formados por muestras reales de uso que se ha llegado a afirmar que "en la actualidad, 'ser lingüista' significa cultivar lingüística de corpus” (ibíd.: 20). El presente trabajo, precisamente, se desarrolla a partir de un corpus oral compuesto por distintas interacciones transcritas en formato CHAT (Codes for the Human Analysis of Transcripts) en las que intervienen niños con y sin síndrome de Asperger (SA).

Partimos del supuesto de que el perfil lingüístico-comunicativo de las personas con SA se caracteriza, entre otras cosas, por mostrar una clara disonancia entre las habilidades de carácter gramatical y las específicamente pragmáticas. Tales discrepancias se han materializado, en diversas investigaciones de corte experimental (cfr. Vulchanova et al., 2012, 2015), en un desempeño comparativamente mejor en tareas centradas en componentes estructurales del lenguaje que en aquellas que implican la comprensión y producción de componentes pragmáticos. En este sentido, son numero- 
sos los autores que descartan que los usuarios con SA muestren problemas asociados a las habilidades gramaticales y léxicas (Norbury, 2004, 2005; Oakhill y Cain, 2012), a diferencia de las habilidades pragmáticas, área en la que se concentraría el mayor número de disfunciones (Rodríguez Muñoz, 2012, 2013).

En sintonía con lo anterior, en el presente estudio, sugerimos la disociación de estos dos ámbitos del lenguaje en el SA, a saber, el gramatical o estructural, y el pragmático o comunicativo. Tal descompensación es la que determina nuestra consideración del SA como déficit pragmático. Consideramos, asimismo, que los resultados tan contrarios en tareas que requieren el procesamiento de elementos formales (en ocasiones, por encima de los valores estándares), frente a aquellas otras en las que intervienen el significado inferencial y el contexto (por debajo de los valores normalizados) son una de las consecuencias derivadas del déficit neurológico de integración de las funciones cerebrales que se les ha atribuido a los sujetos con SA (cfr. Remschmidt, 2004), el cual nos remite inevitablemente a la teoría de la infraconectividad (Just et al., 2004, 2007; Kana et al., 2006; Mason et al., 2008; Mizuno et al., 2011).

De acuerdo con la postura que adoptamos en este trabajo, el SA es, además de una alteración de carácter pragmático, un desorden que puede ser explicado por un déficit al integrar los elementos formales de la lengua (gramática) y los factores contextuales que determinan los intercambios (pragmática). Así pues, el objetivo principal de este trabajo consistirá en sustentar dicha hipótesis a partir del análisis de una serie de categorías de tipo gramatical (complejidad sintáctica y diversidad léxica, cohesión y coherencia), y de otras que son específicamente pragmáticas (principio de cooperación y tareas de edición).

En suma, no esperamos encontrar desventajas significativas en el comportamiento discursivo desde un punto de vista gramatical tras analizar las categorías vinculadas a dicha habilidad en las interacciones orales de los sujetos con SA, si bien prevemos que estos muestren algunas limitaciones al atender a la conducta comunicativa desde una perspectiva puramente pragmática.

\subsection{El síndrome de Asperger, déficit pragmático}

En los últimos años ha sido creciente el interés que ha suscitado el comportamiento comunicativo de las personas con SA y autismo de alto funcionamiento (AAF) -hoy en día existe un amplio consenso al equiparar el SA y el AAF, que comparten la misma sintomatología y tan solo presentan diferencias de grado (cfr. Howlin, 2003)-. En la quinta edición del Manual diagnóstico y estadístico de los trastornos mentales (DSM-5) (Asociación America- 
na de Psiquiatría, 2014), el rótulo trastorno del espectro autista (TEA) abarca el SA junto con cuatro condiciones más (el trastorno autista -o autismo-, el trastorno desintegrativo infantil, el trastorno de Rett y el trastorno generalizado del desarrollo no especificado). Frente a la voluntad integradora que se percibe en relación con estas etiquetas, en la última versión del DSM, se incorpora una nueva, el denominado trastorno de la comunicación social (pragmática), que se define como "una nueva afección que implica dificultades persistentes en los usos sociales de la comunicación verbal y no verbal" (ibíd.: 809).

Llama la atención que en el DSM-5 se unifiquen unos descriptores y que, en cambio, se proponga "una nueva afección”, que, además, guarda una estrecha relación con el SA. Según establece el DSM-5, para que un individuo con el antiguo diagnóstico de SA pueda ser considerado como TEA, debe presentar, por ejemplo, patrones restrictivos de conducta, intereses o actividades, pues, de lo contrario, tendría que ser evaluado como un trastorno de comunicación social (pragmática) (ibíd.: 51).

De acuerdo con la postura adoptada en este y en otros trabajos (cfr. Rodríguez Muñoz, 2012, 2013), el SA se define por las limitaciones pragmáticas en el comportamiento verbal, paraverbal y no verbal (sobre todo, al tratar de trasladar e interpretar la intencionalidad o la fuerza ilocutiva de los actos comunicativos). Asimismo, las dificultades en las relaciones interpersonales que se les atribuyen a los individuos con SA serían, a menudo, una consecuencia más del déficit pragmático que presentan. Este déficit pragmático es compartido, además, tanto por aquellos que pudieron ser diagnosticados como SA, y que hoy podrían serlo como TEA, cuanto por quienes reciban el diagnóstico de trastorno de la comunicación social, acorde con la nueva categorización del DSM-5.

Por último, si quisiéramos relacionar el SA con el concepto de competencia comunicativa, podríamos acudir al modelo de Bachman (1995), para quien la competencia pragmática comprendería, por un lado, la ilocutiva (el análisis de las condiciones que permiten que un enunciado sea o no aceptable) y, por otro, la sociolingüistica (la capacidad de interpretar referencias culturales y el lenguaje figurado, así como la sensibilidad hacia las diferencias de registro, la naturalidad y la variación dialectal). Es decir, el SA se definiría, en esencia, como un déficit de competencia pragmática, por cuanto las principales alteraciones del trastorno se reúnen en torno a la ilocutividad de los enunciados y a los aspectos sociolingüísticos de la comunicación. Más específicamente, se trata de un déficit pragmático primario en el que, parafraseando a Perkins (2004: 13), la capacidad lingüística (fonología, morfología, sintaxis, léxico) se encuentra esencialmente intacta, pero la actuación comunicativa está alterada a causa de una disfunción cognitiva no lingüística (p. ej., función ejecutiva, teoría de la mente, afectividad, etc.). 


\subsection{La teoría de la infraconectividad y el déficit de integración en el espectro autista}

Parece evidente que el cerebro de las personas con TEA y, particularmente, con SA responde de una forma diferente ante determinados tipos de estímulos y tareas en las que está involucrado el lenguaje. Si acudimos a la teoría de la infraconectividad (Just et al., 2004, 2007; Kana et al., 2006; Mason et al., 2008; Mizuno et al., 2011), se sugiere que la conexión entre determinadas regiones cerebrales es inferior en los individuos con TEA, sobre todo, en la banda que comunica las áreas frontales con las áreas corticales posteriores. Asimismo, "los patrones de activación y de sincronización temporal de las redes corticales estarían alterados, favoreciendo las conexiones locales en detrimento de las proyecciones a largas distancias" (Marí, 2014: 22). En este sentido, existiría una relación causal entre los fenómenos anatómicos, fisiológicos (actividad cerebral) y psicológicos, incluidos los lingüísticos.

Dicha infraconectividad entre redes neuronales emplazadas en distintas áreas del cerebro estaría impidiendo el logro en tareas lingüísticas que demandaran un alto nivel de integración. Por ejemplo, en el caso del SA, podría tratarse de tareas en las que estuviera implicada la capacidad inferencial (p. ej., las peticiones indirectas, la comprensión de los dobles sentidos, de las metáforas, de la ironía o del humor) o la adecuación a un cierto grado de formalidad, tecnicidad, etc., según las exigencias del contexto, del ámbito o de los interlocutores. Aunque, de acuerdo con los autores que proponen esta teoría, el procesamiento gramatical de los enunciados respondería, por lo general, a los valores estándares o normalizados, los usuarios con SA presentarían dificultades al tratar de integrarlos en un todo contextual que, en última instancia, sería el responsable de otorgarles un sentido.

Al hablar de infraconectividad, nos estamos refiriendo, en definitiva, al bajo funcionamiento de los circuitos de integración y a la falta de coordinación entre regiones concretas del cerebro; lo que conlleva déficits de carácter cognitivo y lingüístico, que son más perceptibles cuanto mayor es el nivel de integración y abstracción que requieren las tareas de procesamiento. Por ende, las dificultades que manifiestan los sujetos con SA en los procesos de integración gramatical -formas, estructuras- en el contexto pragmático -donde son provistas de sentido- podrían explicarse por un bajo nivel de conexión entre las redes neuronales ubicadas en distintas zonas del cerebro, con patrones de activación y sincronización alterados.

Just et al. (2004) comparan, concretamente, los niveles de activación de las dos áreas típicamente asociadas al lenguaje. A la vista de sus resultados, los sujetos con SA se caracterizarían por mostrar una habilidad superior al procesar las palabras (significantes) de manera aislada, que se 
correspondería con una activación también mayor en el área de Wernicke; pero presentarían dificultades a la hora de integrar tales palabras en una estructura dotada de significado (y, aún más, en un contexto), lo que implicaría una menor activación de las redes neuronales situadas en el área de Broca. En efecto, al procesar el significado en tareas de comprensión de oraciones, la activación del área de Wernicke es mayor en los sujetos con TEA que en los que no presentan esta condición y, a la inversa, el área de Broca muestra una activación inferior en los individuos con TEA que en sus pares sin TEA, como se observa en la figura 1.

FIGURA 1. Mayor activación del área de Wernicke (lóbulo temporal) y menor activación del área de Broca (lóbulo frontal) en sujetos con TEA al realizar tareas de comprensión de oraciones

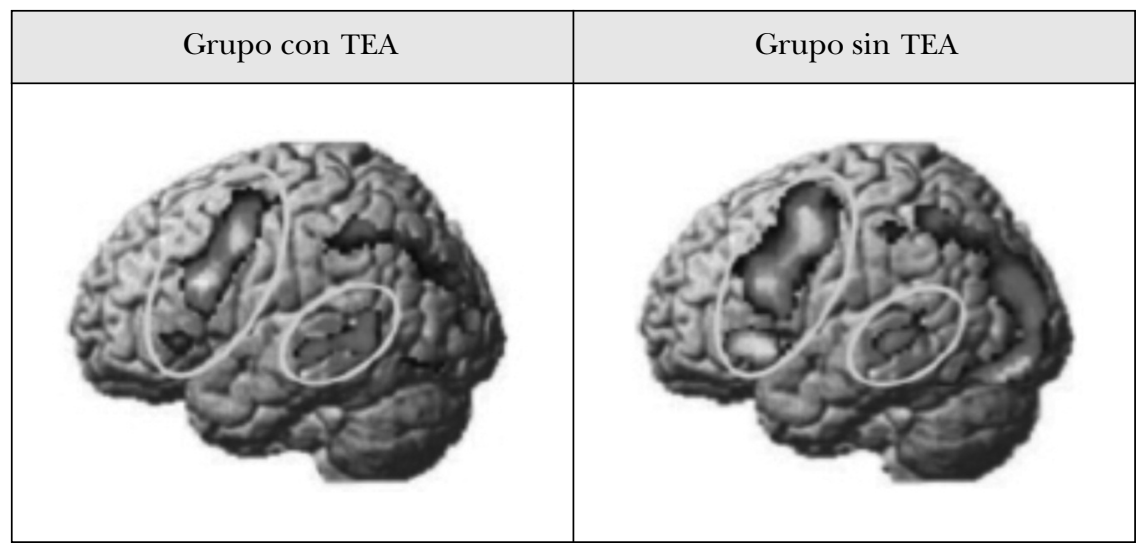

Fuente: Just et al. (2004)

Atendiendo a la organización interna del sistema, tal como señala Ardila (2006), las dificultades que se asocian a los distintos tipos -en su casode afasia apuntan a dos operaciones básicas: la selección (el lenguaje como paradigma) y la secuenciación (el lenguaje como sintagma). Así pues, el déficit de integración que se produce entre el procesamiento de las formas aisladas (significantes) y de su significado contextualizado podría hacerse extensivo a los ejes sintagmático y paradigmático en el SA; es decir, al igual que tiene lugar una descoordinación entre gramática y pragmática, es posible que esta también acaezca entre sintagma y paradigma.

Como explican Gallardo Paúls y Hernández Sacristán (2013: 121) a propósito de los sinónimos, en muchas situaciones de déficit comunicativo, la "capacidad o motivación contextualizadora se ha perdido" o, incluso, puede que nunca haya existido, como ocurriría en el SA, donde -a nuestro 
entender- se carecería “del 'sentido' o el 'para qué' de la operación sinonímica”. Los mismos autores consideran que las dificultades al intentar acceder a significados figurados - por ejemplo, en el SA- se deben, precisamente, a un déficit de integración "entre la esfera referencial del enunciado y la enunciación” (ibíd.: 19). Más adelante, añaden que tal vez podría establecerse un paralelismo entre los déficits de habla no fluente y fluente, y la naturaleza del déficit, con predominio gramatical o pragmático, respectivamente, como es el caso del SA (ibíd.: 139).

Como ya se ha advertido, en este trabajo partimos de la idea de que el SA es un déficit de carácter pragmático. Ello se pone de manifiesto en la descompensación existente entre las habilidades gramaticales -intactas o inalteradas- y las pragmáticas. Tales disonancias tienen su origen en un déficit de integración entre las formas y el significado (particularmente, el inferencial), que encuentra una explicación en la teoría de la infraconectividad.

\section{MARCO TEÓRICO}

La distinción entre habilidades de carácter gramatical, por un lado, y pragmático, por el otro, es deudora de la que se establece en el Protocolo Rápido de Evaluación Pragmática (PREP) (Gallardo Paúls, 2007) -que participa de una concepción amplia y holística de la pragmática- y en el Protocolo Rápido de Evaluación Pragmática-Revisado (PREP-R) (FernándezUrquiza et al., 2015) -su versión más reciente- entre habilidad pragmática de base gramatical y habilidad pragmática específica. Esta diferencia es útil a la hora de identificar la naturaleza de los déficits comunicativos y, lo que es más importante aún, sirve para orientar la posterior intervención, o sea, proporciona información sobre los aspectos lingüísticos en los que se ha de incidir en esta.

\subsection{Habilidades de carácter gramatical}

\subsubsection{Complejidad sintáctica y diversidad léxica}

Como señalan Vulchanova et al. (2012), a pesar de que existen numerosos ejemplos de talentos lingüísticos diagnosticados con SA, dicho talento se limita a la competencia estructural de la lengua -se habla de "ventaja gramatical" o de "fortalezas lingüísticas en los niveles morfológico y sintáctico"-, pues los problemas relacionados con determinados aspectos pragmáticos -por ejemplo, la comprensión del lenguaje figurado o la ca- 
pacidad de inferir a partir del contexto- son persistentes. Estos autores ponen en relación la ya mencionada teoría de la infraconectividad con la disociación existente entre ambas dimensiones, la gramatical y la pragmática, y consideran que la adquisición de la gramática es privilegiada por las conexiones en áreas locales del cerebro, frente a las que se establecen entre áreas más alejadas, que serían la consecuencia de muchas de las disfunciones ligadas al plano pragmático.

Aprovechando que nuestros datos orales se encuentran transcritos en formato CHAT, la utilidad KIDEVAL que incluye el programa CLAN (Computerized Language ANalysis) (MacWhinney, 2000) nos permite obtener, entre otras medidas, dos de las que se han considerado con mayor asiduidad en el análisis del desarrollo gramatical (cfr. MacWhinney, 2008; Ratner y Brundage, 2016): la longitud media de los enunciados (LME) y la diversidad de vocabulario (Dvoc). El primer índice, la LME, convencionalmente se ha expresado en número de morfemas $\left(\mathrm{LME}_{\mathrm{m}}\right)$, si bien a través del programa CLAN puede expresarse también en número de palabras $\left(\operatorname{LME}_{\mathrm{p}}\right)$. En cuanto a la Dvoc, se trata de una alternativa a la tradicional type-token ratio (TTR) (cfr. Malvern y Richards, 1997) y viene determinada por la cantidad de palabras diferentes que son empleadas en el discurso; esto es, cuanto más elevado es el valor Dvoc, mayor será la riqueza léxica y la complejidad textual que este presentará.

\subsubsection{Cohesión (conectividad)}

Fine et al. (1994) llevan a cabo una investigación pionera -se publica poco después de que sea reconocido oficialmente el SA- a propósito de la cohesión discursiva en conversaciones en las que participan sujetos con SA. Aunque su estudio se centra en la deixis y la foricidad, conviene destacar que los resultados del grupo con SA al emplear elementos cohesivos de este tipo fueron muy parecidos a los del grupo sin SA con el que se comparan.

En nuestro caso, atendemos a la conectividad, es decir, prestamos atención a otra clase de elementos cohesivos a través de los cuales el mensaje es concebido como un texto conectado. En concreto, los elementos que analizamos equivalen a los marcadores relacionales (Roulet et al., 1985) o, en la terminología de Martín Zorraquino y Portolés (1999), a los conectores.

Tales partículas poseen un valor sintáctico-proposicional intra- o interoracional (cfr. Briz, 1993) que contribuye a cohesionar el discurso. Mediante estos elementos lingüísticos se establecen relaciones semánticas de distinta clase; en nuestro análisis nos ocupamos de las que expresan adición, opcionalidad, contraste, consecuencia, justificación, aclaración, tiempo, finalidad y condición. 


\subsubsection{Coherencia (superestructuras narrativas)}

Son numerosas las investigaciones en las que se ha demostrado la utilidad de analizar las narraciones orales de poblaciones que presentan algún tipo de déficit con objeto de afinar su caracterización lingüístico-comunicativa; el SA no ha sido ajeno a este hecho (cfr. Rumpf et al., 2012; Rodríguez Muñoz, 2016). Precisamente, la lámina correspondiente a la tarea 2 del apoyo gráfico que empleamos para motivar la interacción de los participantes (cfr. § 3.3), representada en la Figura 2, estaba encaminada a la narración oral.

Para valorar la coherencia en el nivel superestructural, seguimos a Marinkovich (1999), quien adapta el esquema clásico de las fases narrativas propuesto por Adam (1987) para determinar si los hablantes evaluados identifican los acontecimientos que se suceden en el relato a partir de di-

FigURA 2. Tarea 2 del apoyo gráfico destinada a la narración oral

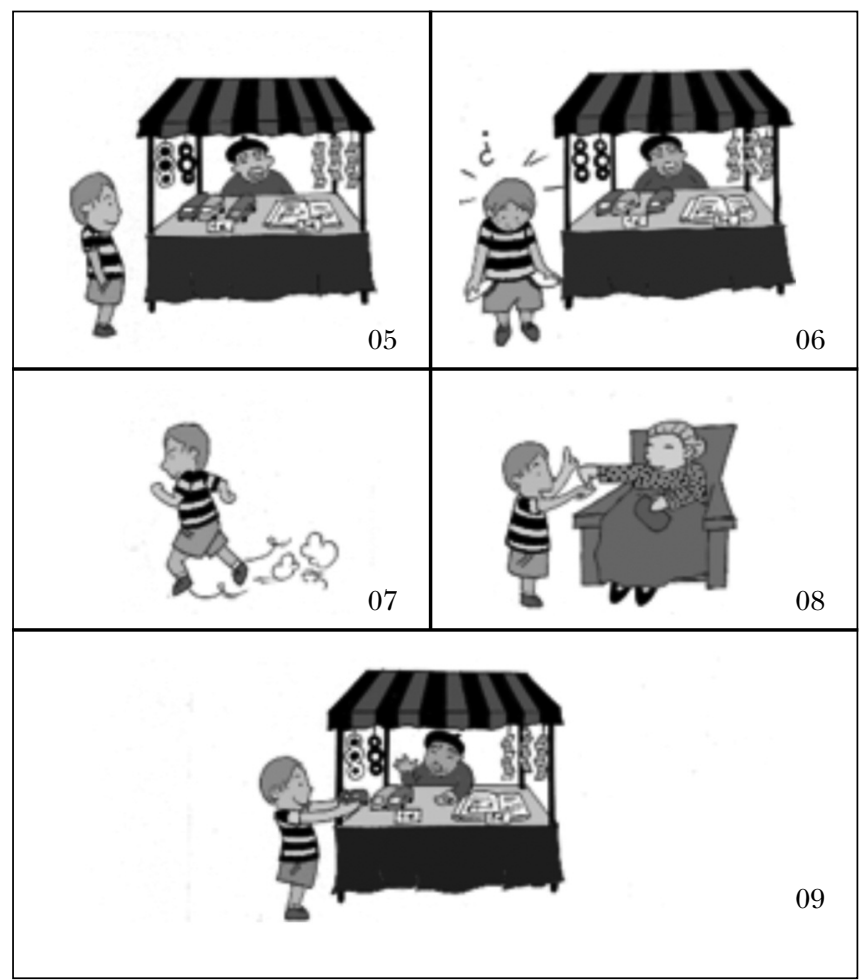

Fuente: Rodríguez Muñoz (2012) 
chas fases. La puntuación que se le asigna a cada fase se define cualitativa y cuantitativamente por los siguientes valores: 4 =óptimo, 3 = satisfactorio, 2 = bajo, y 1 = ausente.

Los valores anteriores se aplican según el grado en que son reconocidos los eventos narrativos de acuerdo con el siguiente prototipo:

(1) Anuncio: un niño llega a un puesto de juguetes.

(2) Inicio: quiere comprarse algo que ha visto.

(3) Complicación: pero se da cuenta de que no lleva dinero.

(4) Reacción: así que sale corriendo a pedir dinero.

(5) Modificación: una mujer mayor, tal vez su abuela, le da dinero.

(6) Final: finalmente, se compra un coche verde de juguete.

\subsection{Habilidades de carácter pragmático}

\subsubsection{Principio de cooperación}

De acuerdo con Grice (1989), en todo intercambio, los interlocutores manifiestan distintos grados de colaboración a la hora de comunicarse. El supuesto pragmático que encierra este acuerdo tácito en la interacción es el denominado principio de cooperación, que se desarrolla, grosso modo, atendiendo al cumplimiento de cuatro máximas: calidad, cantidad, manera y relación.

Según sostienen distintos autores (cfr. Kasher y Meilijson, 1996), los sujetos con TEA presentan dificultades al tratar de aplicar dichas máximas, de modo que la vulneración de alguna de ellas implicaría una falta de cooperación comunicativa. Los problemas al captar las sutilezas de cada una de estas máximas cuando son trasladadas e integradas en distintos contextos parecen encontrarse en la base del fallo pragmático y, a nuestro juicio, son una manifestación más del déficit de integración entre los planos gramatical y pragmático, que puede explicarse a partir de la teoría de la infraconectividad.

Para evaluar el principio de cooperación, recurrimos al ítem 5 del PREP-R (Fernández-Urquiza et al., 2015); concretamente, aplicamos los subítems 5.1 a $5.4^{1}$, que, como refleja la tabla 1 , se marcan positiva o negativamente -también existe la opción de no evaluarlos- según se respeten o no las máximas griceanas en la interacción.

\footnotetext{
${ }^{1}$ En el PREP-R se relaciona el ítem 5.3 (máxima de manera) con la denominada habilidad pragmática de base gramatical, a diferencia de los tres ítems restantes, que se tienen en cuenta al evaluar la habilidad pragmática específica.
} 
TABLA 1. Ítems 5.1 a 5.4 del PREP-R

\begin{tabular}{|c|c|c|c|}
\hline $\begin{array}{c}\text { ¿Se ajusta la conducta verbal del paciente } \\
\text { a las siguientes afirmaciones? }\end{array}$ & Sí & No & $\begin{array}{l}\text { No } \\
\text { eval. }\end{array}$ \\
\hline SUBNIVEL DE INFERENCIAS & & & \\
\hline \multicolumn{4}{|l|}{ 5. Principio de cooperación } \\
\hline \multicolumn{4}{|l|}{ 5.1. La información que proporciona es veraz (calidad) } \\
\hline \multicolumn{4}{|l|}{$\begin{array}{l}\text { 5.2. La información que proporciona no es ni escasa } \\
\text { ni excesiva, siempre teniendo en cuenta } \\
\text { los requerimientos contextuales (cantidad) }\end{array}$} \\
\hline $\begin{array}{l}\text { 5.3. La información se proporciona de manera clara, } \\
\text { ordenada y sin ambigüedades (manera) }\end{array}$ & & & \\
\hline $\begin{array}{l}\text { 5.4. Sus intervenciones tienen relación con el tema } \\
\text { de conversación (relación) }\end{array}$ & & & \\
\hline
\end{tabular}

Fuente: Fernández-Urquiza et al. (2015)

\subsubsection{Tareas de edición}

El concepto tarea de edición, en ocasiones, se ha equiparado a la labor del montaje cinematográfico, en la que el montador desecha tomas falsas y selecciona el material que configura la edición final (Gallardo Paúls y Moreno Campos, 2006). Entre las conductas comunicativas que se asocian a esta noción se incluyen las pausas oralizadas, expresiones (marcadores del discurso, pronombres, etc.) mediante las cuales los hablantes rellenan el silencio (gap-fillers) a la vez que procesan mentalmente y planifican la información lingüística que van a emitir. Las pausas vacías (o silentes) también se consideran tareas de edición cuando, según sugieren Crockord y Lesser (1994), superan los dos minutos.

De acuerdo con lo anterior, distinguiremos, de un lado, pausas oralizadas (hmm) y marcadores metadiscusivos conversacionales (bueno y eh) (Martín Zorraquino y Portolés, 1999) y, de otro, pausas silentes (> $2 \mathrm{~min}$ ) en el discurso oral de los hablantes con SA.

\section{AsPectos MetodolóGicos}

\subsection{Participantes}

Esta investigación forma parte de otra más amplia en la que participaron 40 niños hispanohablantes; 20 de ellos, diagnosticados con SA, y otros 
20, sin SA (Rodríguez Muñoz, 2012). El diagnóstico del primer grupo de participantes fue confirmado por los psicólogos que colaboraron en la recogida de datos, que aplicaron el Social Communication Questionnaire (SCQ) (Rutter, Bailey y Lord, 2003).

Con respecto al grupo de hablantes con SA, 14 sujetos procedían de Asturias (España) y 6 de Valencia (España). Los datos fueron recogidos de forma presencial en ambas zonas geográficas. Las edades cronológicas de estos oscilaban entre los 6 y los 15 años $(M=11,15) ; 19$ de ellos eran niños y solo se incluyó la grabación de una niña ${ }^{2}$. En el momento de la recogida de datos, los participantes no se encontraban bajo tratamiento farmacológico alguno.

Los 20 sujetos sin SA procedían de Almería (España), excepto una niña mexicana. Este grupo estuvo formado por 11 niños y 9 niñas con edades cronológicas comprendidas entre los 12 y los 13 años $(M=12,35)$. Ninguno de los participantes presentaba problemas psicológicos o neurológicos de acuerdo con la información proporcionada por el Departamento de Orientación Psicopedagógica del IES Argar, centro de enseñanza en el que fueron recogidos los datos de manera igualmente presencial.

\subsection{Corpus}

El corpus del que se nutre la presente investigación está formado por dos subcorpus. En el primero se agruparon 20 grabaciones en audio correspondientes a los participantes sin SA, que sumaron 97,7 minutos. El segundo subcorpus estuvo integrado por 20 grabaciones audiovisuales protagonizadas por los sujetos con SA y alcanzó los 149,3 minutos.

Los datos orales fueron transcritos en formato CHAT, aplicando los códigos que se especifican en el Anexo.

\subsection{Instrumento}

Para facilitar la recogida de las muestras orales de los sujetos con y sin SA, basándonos en los materiales propuestos por Monfort y Monfort (2001), diseñamos un apoyo gráfico en el que se trataban contenidos de interés pragmático (Rodríguez Muñoz, 2013, 2014a). Por lo tanto, el propósito principal de este instrumento fue motivar un discurso oral semiorientado a partir de viñetas agrupadas en ocho situaciones diferentes en las que se abordaban aspectos tales como la comprensión de inferencias y de estados anímicos ajenos, el uso de verbos mentalistas, la narración de eventos, el humor o la empatía, entre otros.

${ }^{2}$ Téngase en cuenta que la prevalencia del SA es superior en el sexo masculino. 


\subsection{Procedimiento}

Antes de realizar las grabaciones, se les suministraron las siguientes instrucciones generales a los participantes:

Te voy a mostrar una serie de fichas con viñetas y te haré unas preguntas muy sencillas sobre lo que observas en cada una de ellas. Por ejemplo, te pediré que te pongas en el lugar de algunos personajes o que me cuentes una pequeña historia a partir de los dibujos. Intenta, siempre que puedas, referirte al número de la viñeta que estás comentando.

Seguidamente, se fueron mostrando las distintas láminas con ilustraciones y, al mismo tiempo, se iban planteando las cuestiones que habían de responderse oralmente a partir del apoyo gráfico.

\subsection{Categorías y datos analizados}

Dada la naturaleza diversa y la heterogeneidad de las categorías que son abordadas en este trabajo, basándonos en el PREP (Gallardo Paúls, 2007) y el PREP-R (Fernández-Urquiza et al., 2015), en la tabla 2 se organizan según

TABLA 2. Categorías, subcategorías y datos analizados

\begin{tabular}{|l|l|l|}
\hline \multicolumn{3}{|c|}{ Habilidades de carácter gramatical } \\
\hline \multicolumn{1}{|c|}{ Categorías } & \multicolumn{1}{|c|}{ Subcategorías } & \multicolumn{1}{c|}{ Datos analizados } \\
\hline $\begin{array}{l}\text { Complejidad } \\
\text { sintáctica } \\
\text { y diversidad } \\
\text { léxica }\end{array}$ & $\begin{array}{l}\text { Longitud media del enunciado } \\
\text { por número de palabras }\left(\mathrm{LME}_{\mathrm{p}}\right) \\
\text { y de morfemas }\left(\mathrm{LME}_{\mathrm{m}}\right)\end{array}$ & $\begin{array}{l}\text { Promedio }(M) \text { de los índices } \\
\text { LME y Dvoc calculados } \\
\text { a partir del programa CLAN } \\
\text { (MacWhinney, 2000) }\end{array}$ \\
\hline Cohesión & $\begin{array}{l}\text { Diversidad de vocabulario (Dvoc) } \\
\text { Conectores (marcadores } \\
\text { opcionales) de adición, } \\
\text { consecuencia, justificación, } \\
\text { aclaración, tiempo, finalidad } \\
\text { y condición }\end{array}$ & $\begin{array}{l}\text { Frecuencia absoluta }\left(F_{i}\right) \\
\text { yrecuencia relativa }\left(f_{i}\right)\end{array}$ \\
& $\begin{array}{l}\text { Tokens (diversidad en el } \\
\text { repertorio) }\end{array}$ \\
\hline Coherencia & Superestructuras narrativas & $\begin{array}{l}\text { Nivel de logro (4-1) estimado } \\
\text { a partir de la verbalización } \\
\text { coherente de los eventos } \\
\text { en cada fase de la narración } \\
\text { (tarea 2 del apoyo gráfico) } \\
\text { (cfr. Marinkovich, 1999) }\end{array}$ \\
\hline
\end{tabular}


TABLA 2 (cont.). Categorías, subcategorías y datos analizados

\begin{tabular}{|l|l|l|}
\hline \multicolumn{2}{|c|}{ Habilidades de carácter pragmático } \\
\hline Categorías & \multicolumn{1}{|c|}{ Subcategorías } & \multicolumn{1}{c|}{ Datos analizados } \\
\hline $\begin{array}{l}\text { Principio de } \\
\text { cooperación }\end{array}$ & $\begin{array}{l}\text { Máximas de calidad, cantidad, } \\
\text { manera y pertinencia }\end{array}$ & $\begin{array}{l}\text { Porcentajes }(\%) \\
\text { que determinan el grado } \\
\text { de cumplimiento } \\
\text { o incumplimiento } \\
\text { (Ejemplos orales) }\end{array}$ \\
\hline $\begin{array}{l}\text { Tareas } \\
\text { de edición }\end{array}$ & $\begin{array}{l}\text { Pausas oralizadas y marcadores } \\
\text { metadiscursivos conversacionales }\end{array}$ & $\begin{array}{l}\text { Frecuencia absoluta }\left(F_{i}\right) \text { y } \\
\text { frecuencia relativa }\left(f_{i}\right) \text { de los } \\
\text { marcadores metadiscursivos } \\
\text { Pausas vacías (o silentes) } \\
(>2 \text { min) }\end{array}$ \\
(Ejemplos orales)
\end{tabular}

se asocien a las habilidades de carácter gramatical o pragmático. Además, se indican las subcategorías en las que se centra el análisis, así como los datos concretos que pretendemos analizar.

\section{RESUlTADOS Y DISCUSIÓN}

\subsection{Habilidades de carácter gramatical}

\subsubsection{Complejidad sintáctica y diversidad léxica}

Como muestra la Tabla 3, los valores correspondientes a la LME, tanto en número de palabras $\left(\mathrm{LME}_{\mathrm{p}}\right)$ como de morfemas $\left(\mathrm{LME}_{\mathrm{m}}\right)$, y a la Dvoc son ligeramente superiores en el discurso oral de los participantes con SA que en el de sus pares sin SA; sin embargo, las diferencias no son significativas.

Tabla 3. Promedio (M) de los índices LME y la Dvoc en el discurso oral de los participantes con y sin SA

\begin{tabular}{|c|c|c|c|}
\cline { 2 - 4 } \multicolumn{1}{c|}{} & $\mathbf{L M E}_{\mathbf{p}}$ & $\mathbf{L M E}_{\mathbf{m}}$ & Dvoc \\
\hline Con SA & 9,55 & 12,78 & 63,08 \\
\hline Sin SA & 8,91 & 10 & 58,17 \\
\hline
\end{tabular}


Los datos anteriores relativos a la LME y a la Dvoc son equiparables a los que exponen Wetherell, Botting y Conti-Ramsden (2007) al comparar las interacciones orales de niños con y sin trastorno específico del lenguaje (TEL) con una media de edad equivalente a los 14 años. Al interpretar estos índices, de acuerdo con nuestros resultados, los niños con SA que participan en el estudio no presentan diferencias reseñables en relación con sus homólogos sin SA en términos de complejidad sintáctica o riqueza léxica.

En cuanto a la calidad léxica de los sujetos con SA, conviene advertir que la precisión al seleccionar determinadas palabras que utilizan al describir las imágenes del apoyo gráfico supera en ocasiones las expectativas generadas por la edad. Por ejemplo, en (1), ACR emplea las locuciones pez luna y aleta dorsal, que no son las esperadas en un niño de 6 años, como es su caso.

(1) *INT: <solo $>[<]$ quedan dos (..) mira (.) ¿qué pasa en estas dos viñetas (.) en la veinte y en la veintiuno?

*ACR: parece que: una niña se está asustando porque parece ser que hay $<$ un $>$ [/] un tiburón o un pez luna $<$ en $>$ [/] en $<$ el lago $>$ [ $>]$.

*INT: <vale (.)> [<] bien (.) bien [?] (.) ¿y luego qué ocurre?

*ACR: y ocurre que es un gracioso $\uparrow$ (.) que se había puesto: la típica: aleta dorsal (..) eh@i (...) aquí \&=se señala la espalda.

\subsubsection{Cohesión (conectividad)}

Como se observa en la tabla 4, al comparar los resultados referentes al uso de los conectores en el discurso oral de los participantes con y sin SA, no se producen diferencias significativas ni en términos de frecuencia absoluta $\left(F_{i}\right)$ ni relativa $\left(f_{i}\right)^{3}$ (cfr. Rodríguez Muñoz, 2014b). Solo se produce un predominio cuantitativo en el número de conectores de carácter consecutivo que emplea el grupo de hablantes sin SA, pues, en los demás casos, las cantidades están prácticamente equiparadas, a excepción de las relaciones contrastiva y justificativa, a las que les corresponde un mayor número de conectores en las interacciones orales del grupo con SA.

Desde un punto de vista cualitativo, sin embargo, debemos señalar que el repertorio de elementos lingüísticos de este tipo que es utilizado por los sujetos con SA es más variado. Este hecho se pone de manifiesto en la mayor diversidad de elementos lingüísticos (types) que son empleados para

\footnotetext{
${ }^{3}$ Para calcular el valor $f_{i}$, dividimos el número de conectores entre el número de palabras (tokens) que componen las interacciones orales del grupo de hablantes con y sin SA, que es de 10397 y 8883 respectivamente.
} 
TABLA 4. Los tipos de conectores y su frecuencia absoluta $\left(F_{i}\right)$ y relativa $\left(f_{i}\right)$ en el discurso oral de usuarios con y $\sin S A$

\begin{tabular}{|c|c|c|}
\hline Relación & Con SA & Sin SA \\
\hline Adición & $\begin{array}{l}\text { y (391), además (2), e (1), hasta (1), } \\
\text { incluso (1) }\end{array}$ & $y(401), e(3)$ \\
\hline Subtotal & 396 & 404 \\
\hline Opcionalidad & $o(51)$ & $o(67)$ \\
\hline Contraste & pero (45), aunque (2), sin embargo (1) & pero (14), aunque (1) \\
\hline Subtotal & 48 & 15 \\
\hline Consecuencia & pues (157), entonces (37), así que (3) & $\begin{array}{l}\text { pues }(257) \text {, entonces }(28) \text {, } \\
\text { asi que }(1)\end{array}$ \\
\hline Subtotal & 197 & 286 \\
\hline Justificación & porque $(178)$, es que $(10)$, ya que (5) & porque (103), es que (6) \\
\hline Subtotal & 193 & 109 \\
\hline Aclaración & o sea (4), vamos (3), es decir (1) & - \\
\hline Subtotal & 8 & 0 \\
\hline Tiempo & $\begin{array}{l}\text { después (34), cuando (19), luego (15), } \\
\text { al final (3) }\end{array}$ & $\begin{array}{l}\text { cuando (22), luego (19), } \\
\text { después, }(12), \text { al final (4) }\end{array}$ \\
\hline Subtotal & 71 & 57 \\
\hline Finalidad & para $(27)$ & para (14) \\
\hline Condición & si (23) & si (15) \\
\hline$F_{i}\left(f_{i}\right)$ & $1014(0,0975)$ & $967(0,1089)$ \\
\hline
\end{tabular}

indicar las relaciones semánticas de adición, contraste y justificación entre enunciados, así como en el uso de conectores de aclaración, que están ausentes en las producciones orales de los participantes sin SA.

\subsubsection{Coherencia (superestructuras narrativas)}

La organización de las fases narrativas en las producciones orales de los niños con SA y sus pares sin SA responde en nuestro análisis a los mismos parámetros. De igual modo, al comparar las puntuaciones asignadas a las narraciones orales de unos y otros, como se observa en la tabla 5, no 
se aprecian diferencias cuantitativas (cfr. Rodríguez Muñoz, 2016). Tanto es así que la coincidencia en las puntuaciones de las tres primeras fases de la narración (anuncio, inicio y complicación) es absoluta, y en las restantes, similar.

TABLA 5. Promedio (sobre 4) de las puntuaciones asignadas en cada fase narrativa en las producciones orales de los participantes con y sin SA

\begin{tabular}{|l|c|c|c|c|c|c|}
\cline { 2 - 7 } \multicolumn{1}{c|}{} & Anuncio & Inicio & Complicación & Reacción & Modificación & Final \\
\hline Con SA & 2,55 & 2,95 & 3,85 & 3,2 & 2,85 & 3,65 \\
\hline Sin SA & 2,55 & 2,9 & 3,85 & 3,5 & 2,25 & 3,9 \\
\hline
\end{tabular}

Las puntuaciones precedentes se traducen cualitativamente en un nivel de logro satisfactorio al evaluar la competencia narrativa oral de ambos grupos, que, en este caso, se concreta en el reconocimiento de los eventos que se suceden en la escena gráfica que motiva el relato oral.

\subsection{Habilidades de carácter pragmático}

\subsubsection{Principio de cooperación}

Los resultados obtenidos tras la evaluación de los ítems 5.1 a 5.4 del PREP-R (Fernández-Urquiza et al., 2015) en los intercambios comunicativos en los que intervienen nuestros participantes con SA, como muestra la figura 3, revelan que:

a) la máxima más vulnerada es la de cantidad -en el $90 \%$ de las interacciones orales se produce alguna transgresión en este sentido-;

b) la máxima de manera es vulnerada en el $50 \%$ de las ocasiones;

c) en un $40 \%$, se produce alguna infracción relacionada con la máxima de relación; y

d) tan solo se incumple la máxima de calidad en el $10 \%$.

Debemos llamar la atención sobre el hecho de que el incumplimiento de la máxima de cantidad lleva aparejado, con frecuencia, el de las máximas de pertinencia y manera, ya que el exceso informativo, a veces, implica que se produzcan digresiones temáticas (falta de relevancia) y, al mismo tiempo, se contraviene una propiedad asociada a la máxima de manera, la brevedad, tal como se aprecia en (2) cuando interviene BFG. La vulnera- 
FIGURA 3. Grado de cumplimiento e incumplimiento (\%) de las máximas conversacionales en las interacciones orales de los participantes con SA

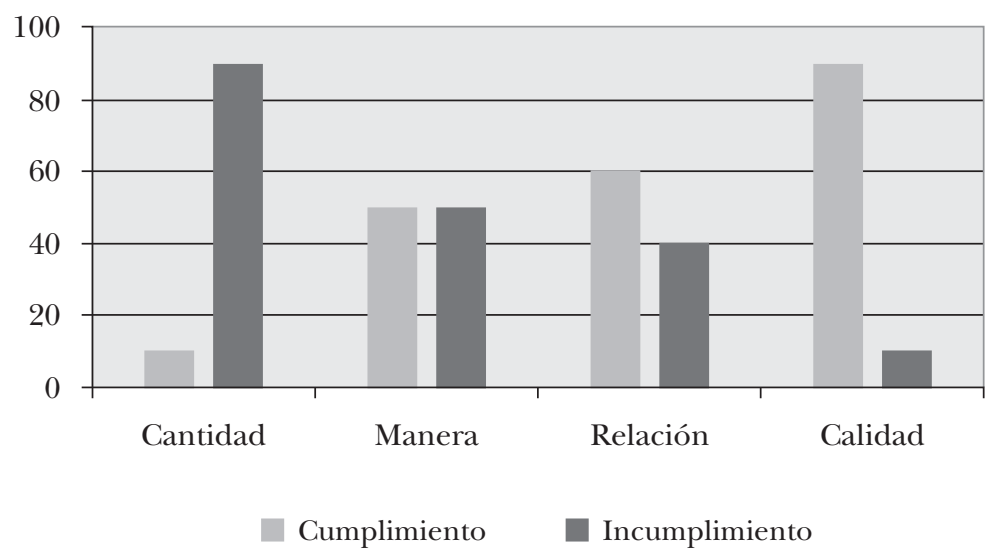

ción de la máxima de cantidad por exceso de datos, además, puede provocar un efecto logorreico que ha sido percibido de forma esporádica y poco representativa en personas con diagnóstico de SA (cfr. Rodríguez Muñoz, 2009).

(2) *INT: vale (.) ¿crees que sería posible que toda esa gente se dirigiese al mismo sitio (..)?

*BFG: eso (.) hay algunas probabilidades de que [//] bueno (.) de que vaya a ese sitio tod (.) todas juntas y s (.) jay! (.) mira (.) hay: una posibilidad de que primero desde es [//] desde la señora de (.) acalorada $<$ con $>[/]$ con el hombre de la compra haya una pequeña posibilidad (.) una diminuta posibilidad $\&=$ aprieta el pulgar y el índice de la mano izquierda de que vayan al mismo sitio y (.) sin embargo (.) sus expresiones son diferentes (.) lo que quiere decir (.) no sé (.) otra cosa (..).

En (3), en cambio, consideramos que en la intervención de JIS se vulnera la máxima de cantidad por defecto informativo en vez de por exceso, como ocurría en (1).

(3) *INT: ¿crees que es posible que toda esta gente [=! JIS tose] se estuviera dirigiendo al mismo sitio?

*JIS: $\quad$ hm:@i (.) creo que no. 
*INT: por ejemplo (.) ¿podrían ser todos vecinos que vivieran en el mismo edificio (..)?

*JIS: ¿ ¿el mismo edificio? (.) ff@i $()<.<$ puede ser $>[=$ ! tono bajo $]>[>]$.

*INT: < <podrían ser $>[<]$ todos vecinos $(.$.$) ?$

*JIS: ¿'todos?

*INT: uhhum@i (.) <¿podría ser?> [>].

*JIS: $\quad<$ pue:s $>[<](.$.$) creo que no.$

Otras veces, la falta de atención o la distracción del hablante es la que condiciona la vulneración de la máxima de pertinencia. Por ejemplo, en (4) APC no dispone de ningún elemento gráfico sobre el que apoyarse en las viñetas que describe para deducir que al personaje que aparece en ellas -un joven sentado en un banco- van a organizarle una fiesta de cumpleaños. Asimismo, los sonidos que emite y las palabras que pronuncia en inglés no contribuyen en modo alguno a la cooperación comunicativa, ya que son completamente inoportunas e irrelevantes.

(4) *INT: +, escucha (.) ¿crees que es posible que toda esta gente se esté di (.) dirigiendo al mismo lugar?

*APC: a:m (.) creo que: sí [=! ralentiza el ritmo] porque creo que le [//] porque creo que le van a organizar una fiesta + /.

*INT: vale.

*APC: +, o: o algo (.) o van a visitarle para su cumpleaños y él le ha invitado $()<$. pi:on@o $>[>] \&=$ emite de nuevo un sonido y señala a cámara.

*INT: $<y()>.[<]$ por ejemplo (.) ¿podrían ser vecinos del mismo edificio?

*APC: xxx power@s:eng (.) shower sound@s:eng [>].

*INT: Álvaro (.) Álvaro [<].

*APC: ¿eh? (.) vaya.

*INT: ¿podrían ser vecinos del mismo edificio?

*APC: em (.) creo que sí.

Por último, en (5) consideramos que se produce la vulneración de la máxima de manera, pues BFG no rehúye la ambigüedad al responder al entrevistador y utiliza un símil inventado para expresar que se pondría furioso si le gastaran una broma.

(5) *INT: vale (.) ¿cómo te sentirías tú si te gastan una broma así (.)?

*BFG: ay (.) ¿para qué mentirte? (.) me sentiría como un oso polar en peligro de extinción $[=!$ se ríe] (.).

*INT: ¡como un oso polar en peligro de extinción! (.) ¿y eso qué quiere decir?

*BFG: que me sentiría como una fiera $\uparrow$. 


\subsubsection{Tareas de edición}

Al comparar el número de pausas oralizadas $(\mathrm{hmm})$ y de marcadores metadiscursivos (bueno y eh) en los subcorpus de hablantes con y sin SA, comprobamos que existe un claro predominio, como muestra la tabla 6 , al usar esta clase de tareas de edición en los turnos en los que intervienen los individuos con SA.

\section{TABLA 6. Frecuencia absoluta $\left(F_{i}\right)$ y relativa $\left(f_{i}\right)$ de las pausas oralizadas y los marcadores metadiscursivos en la interacción oral de los usuarios con y sin $S A$}

\begin{tabular}{|c|c|c|}
\cline { 2 - 3 } \multicolumn{1}{c|}{} & Con SA & Sin SA \\
\hline & bueno $(81), h m m(54)$, eh $(50)$ & hmm $(60)$, eh $(11)$, bueno $(6)$ \\
$\boldsymbol{F}_{i}\left(f_{i}\right)$ & $185(0,2083)$ & $77(0,0074)$ \\
\hline
\end{tabular}

De los marcadores metadiscursivos analizados, el que más asiduamente emplean los hablantes con SA es bueno; al mismo tiempo, contrasta el hecho de que este sea el menos productivo en las interacciones del grupo sin SA. En los intercambios comunicativos, el marcador bueno es polifuncional; por un lado, puede actuar como un continuador o un reformulador y, por otro, puede servir para expresar acuerdo o, incluso, asociado a una pronunciación enfática, desacuerdo (cfr. Briz, Pons y Portolés, 2008).

Para ilustrar la función que aquí nos interesa, el contenido relacional de bueno en (6) es de carácter continuativo, pero no solo contribuye a la ilación, sino que además se relaciona con la función metadiscursiva por cuanto permite resolver los problemas al producir un discurso que no ha podido ser planificado (cfr. López Serena y Borreguero, 2010) -también con carácter metadiscursivo, VGC recurre a la interjección hmm en el fragmento reproducido-.

(6) *INT: muy bien (.) ¿y este (.)?

*VGC: hay un hombre que: (.) tsh@i está enfadado < porque hay una noticia que: $>$ [=! se acerca el micrófono] [/] (.) bueno porque hay una noticia hmm@i que: hmm@i no le gusta [\% viñeta 11$]$.

En (7) recogemos un ejemplo en el que otro hablante con SA, AFB, emplea reiteradamente la marca $e h$ y acaba planteándole al entrevistador una pregunta que funciona como acto de enlace proyectivo y le sirve para conseguir más tiempo antes de responder. 
(7) *INT: ¿crees que podría tratarse de cuatro niños que estuvieran viendo lo mismo en la tele?

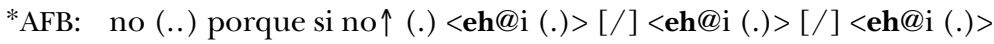
[/] eh@i (.) ¿te lo digo por qué?

*INT: ¿por qué?

*AFB: porque <este $>[/]<$ este está> [///] si <estaría $>$ [*] gritando $\uparrow$ (.) no pueden sentir las mismas emociones por una sola película.

$\mathrm{Al}$ analizar los tipos de pausas vacías o silentes que sobrepasan los dos minutos y su variabilidad funcional a partir del modelo propuesto por Bañón y Requena (2010) (cfr. Rodríguez Muñoz, 2015a), concluimos que la función más habitual que le corresponde a esta clase de pausa en la interacción oral de los niños con SA es la facilitadora y, más específicamente, la que se relaciona con las demandas de planificación al producir el discurso. De hecho, las pausas de más de dos minutos suelen aparecer antes, después o alrededor de las pausas oralizadas y de los marcadores metadiscursivos que hemos señalado antes, como sucede en (8) en el turno de PGM; lo que les permite a los hablantes ganar doble tiempo para procesar la información antes de emitirla.

(8) *INT: ¿catorce [=! se escucha ruido ambiente] (.)?

*PGM: hmm@i (7.) pues que está escuchando música tranquilamente.

En síntesis, el predominio de tareas de edición en las interacciones de los participantes con SA es sintomático, a nuestro juicio, de las mayores demandas de planificación durante la elaboración del discurso que estos presentan en comparación con el grupo de hablantes sin SA.

\section{ConClusión}

Los resultados de este trabajo apoyan los de otros estudios que han determinado que el perfil lingüístico-comunicativo de las personas con SA se caracteriza por las descompensaciones que acontecen entre las habilidades de carácter gramatical, por un lado, y las de carácter pragmático, por otro. Creemos que tal desequilibrio es la consecuencia de un déficit de integración entre el nivel estructural de la lengua y el plano comunicativo, entre las formas lingüísticas y sus posibilidades significativas, entre lo explícito y lo implícito, entre las operaciones sintagmáticas y las paradigmáticas. En este sentido, la teoría de la infraconectividad, según la cual las conexiones de las redes neuronales que median entre distintas zonas del cerebro no se ajustarían a los patrones típicos, nos proporciona una explicación plausible, que, además, nos permitiría tender un puente entre el cerebro y el comportamiento lingüístico de los sujetos con este TEA. 
Tal como preveíamos, el análisis de las categorías que asociamos a las habilidades de carácter gramatical que se ponen en juego en el discurso oral (complejidad sintáctica y diversidad léxica, cohesión y coherencia) nos hace mantener que, desde este punto de vista, el perfil lingüístico-comunicativo de los hablantes con SA no difiere apenas del que se observa en sus pares sin SA. Al contrario, tras examinar las categorías que vinculamos a las habilidades de carácter propiamente pragmático (principio de cooperación y tareas de edición), confirmamos que se producen alteraciones en este ámbito. Esta oposición nos lleva a insistir en la naturaleza pragmática de las disfunciones que se manifiestan en el comportamiento discursivo oral de las personas con SA; en términos de Perkins (2004), se trataría de un déficit pragmático primario.

De acuerdo con lo anterior, en el perfil lingüístico-comunicativo de estos usuarios, el principio de corrección entra en conflicto con el de adecuación sociolingüística, así como con el de cooperación. Es decir, frente a una "normalidad" o superioridad en el nivel estructural, comprobamos que los sujetos con SA exhiben claras dificultades en el dominio pragmático. Conviene advertir que el alto grado de corrección lingüística que prematuramente alcanzan no siempre puede ni debe interpretarse como una virtud social, pues la permanencia en un mismo nivel de habla o registro, por ejemplo, suele implicar la falta de adecuación al contexto y a los interlocutores por parte de quienes presentan SA. Tal inadaptación es la que condiciona, a menudo, el fracaso en las relaciones sociales e interpersonales.

Como señala Bañón (2007: 190), la repercusión de ciertos síndromes, trastornos o enfermedades "sobre los usos lingüísticos ha sido frecuente objeto de estudio por parte de psicolingüistas, biolingüistas, logopedas, fonetistas y analistas del discurso, por ejemplo". Este hecho ha sido más que notorio en el SA, que se ha convertido en un espacio de confluencia e integración de enfoques disciplinares y que se ha beneficiado indudablemente de ello. A los ejemplos que figuran en la nómina anterior se suman otros especialistas como los didactas de la lengua (cfr. Rodríguez Muñoz, 2015b), pues, como propone Yúfera (1993, en Cordero, 2013: 35), a partir de la idea de intervención se ha de facilitar la "retroalimentación entre los conocimientos de los terapeutas del lenguaje y los profesores de lenguas $[\ldots]$, basada precisamente en ese vínculo intervención o mediación educativa $\leftrightarrow$ intervención o reeducación terapéutica".

En definitiva, a través del presente estudio, queremos reivindicar una mayor conexión y permeabilidad de las ciencias del lenguaje con otras disciplinas interesadas en la salud y la enfermedad. De esta manera, como subraya Bañón (2011), podremos alcanzar descripciones e interpretaciones mucho más enriquecedoras de temas como el que aquí hemos abordado. 


\section{BIBLIOGRAFÍA}

Adam, J. M. (1987): “Types de séquences textuelles élémentaires”, Pratiques, 56, págs. 54-79.

ARdilA, A. (2006): "Orígenes del lenguaje: un análisis desde la perspectiva de las afasias”, Revista de Neurología, 43, págs. 690-698.

ASOCIACIÓN AMERICANA DE PSIQUiATRÍA (2014): Manual diagnóstico y estadístico de los trastornos mentales (DSM-5), Arlington, VA, Asociación Americana de Psiquiatría.

BACHMAN, L. (1995): "Habilidad lingüística comunicativa", en M. Llobera (coord.), Competencia comunicativa. Documentos básicos en la enseñanza de lenguas extranjeras, Madrid, Edelsa, págs. 105-127.

BAÑón, A. M. (2007): "Las enfermedades raras y su representación discursiva. Propuestas para un análisis crítico", Discurso \& Sociedad, 1/2, págs. 188-229.

BAÑón, A. M. (2011): "Comunicación, discurso y salud", en J. Fornieles, S. Requena y A. M. Bañón (eds.), Lenguaje, comunicación y salud, Sevilla, Arcibel, págs. $13-57$.

Bañón, A. M. y Requena, S. (2010): "Pausa y descortesía en el debate políticoelectoral”, Español Actual, 94, págs. 9-46.

BRIZ, A. (1993): "Los conectores pragmáticos en español coloquial (I): su papel argumentativo", Contextos, XI, págs. 145-188.

Briz, A., Pons, S. y Portolés, J. (coords.) (2008): Diccionario de partículas discursivas del español, disponible en: <www.dpde.es $>$.

Cordero, E. (2013): Pragmática infantil y enseñanza de español para niños, Madrid, Arco/Libros.

Crockford, C. y Lesser, R. (1994): "Assessing functional communication in aphasia: clinical utility and time demands of three methods", European Journal of Disorders of Communication, 29, págs. 165-182.

FERNÁNDEZ PÉREZ, M. (coord.) (2014): Lingüistica y déficit comunicativos, Madrid, Síntesis.

Fernández-Urquiza, M., Díaz Martínez, F., Moreno Campos, V., Lázaro LópezVillaseñor, M. y Simón LóPez, T. (2015): PREP-R. Protocolo Rápido de Evaluación Pragmática Revisado, Valencia, Universidad de Valencia.

Fernández-Urquiza, M. y Gallardo Paúls, B. (2015): "Adaptación del formato CHAT para la transcripción y codificación de los datos de lenguaje patológico del corpus PerLA", Oralia, 18, págs. 81-102.

Fine, J., Bartolucci, G., Szatmari, P. y Ginsberg, G. (1994): "Cohesive discourse in pervasive developmental disorders", Journal of Autism and Developmental Disorders, 24, págs. 315-329.

Gallardo Paúls, B. (2007): Pragmática para logopedas, Cádiz, Universidad de Cádiz.

Gallardo Paúls, B. y Hernández Sacristán, C. (2013): Lingüística clínica. Un enfoque funcional sobre las alteraciones del lenguaje, Madrid, Arco/Libros. 
Gallardo Paúls, B. y Moreno Campos, V. (2006): "Evolución de la pragmática en un caso de afasia de Broca severa", Revista de Logopedia, Foniatría y Audiología, 26, págs. 188-203.

GRICE, H. P. (1989): Studies in the way of words, Cambridge, MA, Harvard University Press.

Howlin, P. (2003): "Outcome in high-functioning adults with autism with and without early language delays: implications for the differentiation between autism and Asperger syndrome", Journal of Autism and Developmental Disorders, 33, págs. 3-13.

Just, M. A., Cherkassky, V. L., Keller, T. A. y Minshew, N. J. (2004): "Cortical activation and synchronization during sentence comprehension in high-functioning autism: evidence of underconnectivity”, Brain, 127, págs. 1811-1821.

Just, M. A., Cherkassky, V. L., Keller, T. A., Kana, R. K. y Minshew, N. J. (2007): "Functional and anatomical cortical underconnectivity in autism: evidence from an FMRI study of an executive function task and corpus callosum morphometry”, Cerebral Cortex, 17, págs. 951-961.

Kana, R. K., Keller, T. A., Cherkassky, V. L., Minshew, N. J. y Just, M. A. (2006): "Sentence comprehension in autism: thinking in pictures with decreased functional connectivity", Brain, 129, págs. 2484-2493.

Kasher, A. y MeIljJSOn, S. (1996): "Autism and pragmatics of language”, Incontri Cita Aperta, 4, págs. 37-54.

López Serena, A. y Borreguero Zuloaga, M. (2010): "Los marcadores discursivos y la variación lengua hablada vs. lengua escrita”, en Ó. Loureda y E. Acín (eds.), Los estudios sobre marcadores del discurso en español, hoy, Madrid, Arco/Libros, págs. 415-495.

MacWhinney, B. (2000): The CHILDES Project: Tools for Analyzing Talk, 3. ed., Mahwah, NJ, Lawrence Erlbaum Associates.

MACWHINnEY, B. (2008): "Enriching CHILDES for morphosyntactic analysis", en H. Behrens (ed.), Corpora in Language Acquisition Research: History, Methods, Perspectives, Ámsterdam/Filadelfia, John Benjamins, págs. 165-198.

Malvern, D. D. y RichaRds, B. J. (1997): "A new measure of lexical diversity", en A. Wray y A. Ryan (eds.), Evolving models of language, Clevedon, Multilingual Matters, págs. 58-71.

MARí, S. (2014): Valoración antropométrica y nutricional en niños con trastorno del espectro autista, tesis doctoral, Valencia, Universidad de Valencia.

MARINKOVICH, J. (1999): "Una propuesta de evaluación de la competencia textual narrativa”, Revista Signos, 32, págs. 121-128.

Martín Zorraquino, M. A. y Portolés, J. (1999): "Los marcadores del discurso", en I. Bosque y V. Demonte (dirs.), Gramática descriptiva de la lengua española, Madrid, Espasa-Calpe, tomo 3, págs. 4051-4213.

Mason, R. A., Williams, D. L., Kana, R. K., Minshew, N. J. y Just, M. A. (2008): "Theory of Mind disruption and recruitment of the right hemisphere during narrative comprehension in autism”, Neuropsychologia, 46, págs. 269-280.

Mizuno, A., Liu, Y., Williams, D. L., Keller, T. A., Minshew, N. J. y Just, M. A. (2011): "The neural basis of deictic shifting in linguistic perspective-taking in highfunctioning autism”, Brain, 134, págs. 2422-2435.

Monfort, M. y MONFort, I. (2001): En la mente, Madrid, Entha Ediciones. 
NORBURY, C. F. (2004): "Factors supporting idiom comprehension in children with communication disorders", Journal of Speech, Language, and Hearing Research, 47, págs. 1179-1193.

Norbury, C. F. (2005): "The relationship between theory of mind and metaphor: evidence from children with language impairment and autistic spectrum disorders", British Journal of Developmental Psychology, 23, págs. 383-399.

OAKHILL, J. y CAIN, K. (2012): "The precursors of reading ability in young readers: evidence from a four-year longitudinal study”, Scientific Studies of Reading, 16, págs. 91-121.

Perkins, M. R. (2004): Pragmatics and communication disorders. Cambridge, Cambridge University Press.

Ratner, N. B. y Brundage, S. B. (2016): A Clinician's Complete Guide to CLAN and PRAAT, recuperado el 6 de octubre de 2016, de <http://childes.psy.cmu.edu/ manuals/Clin-CLAN.pdf $>$.

REMSCHMidT, H. (2004): “Asperger's syndrome: a pervasive developmental disorder characterized by an integration deficit of cerebral functions", en $\mathrm{H}$. Remschmidt y M. Belfer (eds.), 16th World Congress of the International Association for Child and Adolescent Psychiatry and Allied Professions, Berlín, Springer, pág. 96.

RodRíGuez Muñoz, F. J. (2009): Síndrome de Asperger. Materiales y aproximación pragmalingüistica, Valencia, Universidad de Valencia.

RODRÍGUEZ MuÑoz, F. J. (2012): Los déficits pragmáticos en el discurso oral de niños con sindrome de Asperger, tesis doctoral, Almería, Universidad de Almería.

RodríGuez MuÑoz, F. J. (2013): Evaluación pragmática de niños con síndrome de Asperger, Múnich, Lincom.

RodríGuez MuÑoz, F. J. (2014a): Corpus oral de hablantes con desarrollo típico y síndrome de Asperger, Berlín, Logos Verlag.

RodríGuez MuÑoz, F. J. (2014b): “Discourse markers and modal expressions in speakers with and without Asperger syndrome: a pragmatic-perceptive approach”, Research in Language, 12, págs. 1-25.

Rodríguez MuÑoz, F. J. (2015a): "The pausative pattern of speakers with and without high-functioning autism spectrum disorder from long silences", Pragmatics. Quarterly Publication of the International Pragmatics Association, 25, págs. 229-249.

Rodríguez MuÑoz, F. J. (2015): "Reflexiones teóricas sobre el carácter interdisciplinario de los déficits comunicativos: la combinación de enfoques y su interés en el ámbito de la didáctica de la lengua”, Lengua y Habla, 19, págs. 188-208.

RodRíGUEZ MuÑoz, F. J. (2016): "Evaluación de la competencia semiótico-discursiva a partir de las narraciones orales de niños con y sin síndrome de Asperger: superestructuras textuales y modelos mentales”, Signa, 25, págs. 959-985.

Roulet, E., Auchlin, A., Moeschler, J., Rubattel, C. y Schelling, M. (1985): L'articulation du discours en français contemporain, Berna, Peter Lang.

Rumpf, A. L., Kamp-Becker, I., Becker, K. y Kauschke, C. (2012): "Narrative competence and internal state language of children with Asperger syndrome and ADHD”, Research in Developmental Disabilities, 33, págs. 1395-1407. 
Rutter, M., Bailey, A. y Lord, C. (2003): The Social Communication Questionnaire, Los Ángeles, CA, Western Psychological Services.

Vulchanova, M., Saldaña, D., Chahboun, S. y Vulchanov, V. (2015): "Figurative language processing in atypical populations: the ASD perspective", Frontiers in Human Neuroscience, 9, págs. 1-11.

Vulchanova, M., Talcott, J. B., Vulchanov, V., Stankova, M. y Eshuis, H. (2012): "Morphology in autism spectrum disorders: local processing bias and language", Cognitive Neuropsychology, 29, págs. 584-600.

Wetherell, D., Botting, N. y Conti-Ramsden, G. (2007): "Narrative in adolescent specific language impairment (SLI): a comparison with peers across two different narrative genres", International Journal of Language \& Communication Disorders, 42, págs. 583-605. 


\section{APÉNDICE}

A continuación, se resume la simbología en formato CHAT que se emplea en las transcripciones ${ }^{4}$.

\begin{tabular}{|c|c|c|}
\hline Código & \multicolumn{2}{|c|}{$\begin{array}{r}\text { SigNIFICADO } \\
\end{array}$} \\
\hline$\uparrow$ & Entonación ascendente & \multirow{3}{*}{$\begin{array}{l}\text { Solo en casos en que se vio- } \\
\text { lenten los patrones melódicos } \\
\text { esperables en el enunciado }\end{array}$} \\
\hline$\downarrow$ & Entonación descendente & \\
\hline$\rightarrow$ & Entonación suspendida & \\
\hline$@ \mathrm{i}$ & \multicolumn{2}{|l|}{ Interjección } \\
\hline$@_{S}$ & \multicolumn{2}{|l|}{ Cambio a segunda lengua } \\
\hline$@_{0}$ & \multicolumn{2}{|l|}{ Onomatopeya } \\
\hline$@ \mathrm{~d}$ & \multicolumn{2}{|c|}{ Forma específica de variedad o registro } \\
\hline www & \multicolumn{2}{|c|}{ Fragmento de conversación no transcrito } \\
\hline $\mathrm{xxx}$ & \multicolumn{2}{|c|}{ Habla ininteligible } \\
\hline [?] & \multicolumn{2}{|l|}{ Transcripción dudosa } \\
\hline $\begin{array}{llll}(.) & (. .) & (\ldots) & (4 .)\end{array}$ & \multicolumn{2}{|c|}{$\begin{array}{l}\text { Pausas de menor a mayor duración o de duración } \\
\text { medida en segundos }\end{array}$} \\
\hline vocal: & \multicolumn{2}{|c|}{ Alargamiento vocálico } \\
\hline$[/-]$ & \multicolumn{2}{|l|}{ Reinicio } \\
\hline$[/]$ & \multicolumn{2}{|l|}{ Repetición } \\
\hline sílaba^sílaba & \multicolumn{2}{|l|}{ Pronunciación silabeada } \\
\hline$[=!$ texto $]$ & \multicolumn{2}{|l|}{ Información paralingüística } \\
\hline$\&=$ & \multicolumn{2}{|l|}{ Acciones simultáneas } \\
\hline$+/ /$. & \multicolumn{2}{|l|}{ Autointerrupción } \\
\hline$+/$. & \multicolumn{2}{|c|}{ Interrupción ajena } \\
\hline,+ & \multicolumn{2}{|c|}{ Autoterminación del turno tras interrupción } \\
\hline$[>]$ & \multicolumn{2}{|c|}{ Solapamiento sigue } \\
\hline$[<]$ & \multicolumn{2}{|l|}{ Solapamiento precede } \\
\hline
\end{tabular}

${ }^{4}$ Estas convenciones fueron planteadas en el marco del proyecto de investigación "Ampliación y adaptación del corpus PerLA de datos clínicos en el marco del proyecto internacional CHILDES/Talkbank: perfiles pragmáticos y propuestas de intervención" (FFI2012-39325C03-01). La versión completa y la descripción pormenorizada de la simbología que integra este sistema de codificación puede consultarse en Fernández-Urquiza y Gallardo Paúls (2015). 